\title{
Dejar: entre verbo causativo y verbo de control
}

\author{
Enghels, Renata / Eugeen, Roegiest (Ghent University)
}

Es bien sabido que la marcación del argumento causado en la construcción factitiva causativa es bastante compleja. Al mismo tiempo, la investigación sobre la factitiva suele concentrarse sobre todo en la estructura de hacer con infinitivo, que sirve de modelo a la construcción en general, y los investigadores suelen prestar poca atención a la causación negativa expresada mediante dejar. El estudio actual, basado en un análisis de corpus detallado, persigue la meta de llenar este hueco concentrándose en la interfaz sintaxis-semántica de la factitiva con dejar. El análisis multifactorial que tiene en cuenta tanto la polisemia del verbo principal como los rasgos semánticos de los constituyentes principales, muestra que son los mismos parámetros que influyen en (a) la selección entre un complemento infinitivo y la completiva, (b) la posición del causado nominal y (c) el caso acusativo o dativo del causado pronominal.

It is generally known that the morphosyntactic marking of the causee in the causative factitive construction is rather complex. Moreover, most investigations of the factitive focus on the causative verb hacer ('to do'), which serves as a model for the construction in general, and linguists have paid little attention to related verbs such as dejar ('to let'). This study, based on a detailed corpus analysis, seeks to fill up this gap by centering on the syntax-semantics interface in the factitive expressing negative causation with dejar. A multifactorial analysis, taking into account both the polysemy of the main verb as well as the semantic characteristics of the main constituents of the construction, shows that the same parameters influence on (a) the choice between an infinitive complement or a que-clause, (b) the position of the nominal causee and (c) the accusative or dative case of the pronominal causee.

\section{Introducción}

La construcción factitiva constituye un complejo bipredicativo que comporta dos eventos: un evento causativo cuyo sujeto es el argumento causante del evento complejo (S1) y un evento causado cuyo sujeto es el argumento causado (S2). Se suele distinguir entre una causación 'positiva', con hacer como semi-auxiliar, y una causación 'negativa', con dejar como semiauxiliar.

La investigación sobre la construcción factitiva suele concentrarse sobre todo en la construcción 'hacer + infinitivo' que sirve de modelo a la construcción en general y presta relativamente poca atención a la causación negativa expresada mediante dejar. No obstante, desde una perspectiva semántico-cognitiva ambos verbos representan dos tipos de causación fundamentalmente diferentes, lo que sin duda alguna repercute en el comportamiento sintáctico de las factitivas que forman. Además trataremos de demostrar que el verbo dejar no pertenece a la misma categoría léxica, ni presenta el mismo estatuto gramatical que hacer. 
En términos muy generales (cf. Verhagen / Kemmer 1997, Talmy 2000), la causación negativa supone que el causante se presenta como una entidad con menos control y responsabilidad sobre la ejecución del evento de los que tiene el causante en una causación positiva. Con todo conviene matizar esta postura, teniendo en cuenta la polisemia variable de dejar. Entre los pocos estudios dedicados a esta riqueza semántica destacan las publicaciones de Silva (1997, 2001, 2007), según las cuales se distinguen por lo menos tres matices diferentes de deixar en portugués cuando se combina con un infinitivo. Maldonado (2007) mostró que los tres núcleos semánticos 'no oponerse' (1), 'permitir' (2) y 'soltar' (3) se aplican también al dejar español:

(1) El profesor de gimnasia también estaba, ya se lo explicarían todo en cuanto llegase. Sin saber muy bien por qué dejó que Chitón entrara en el coche. (CREA: Cohen E., Muerte Dulce, 1993)

(2) ¿Me dejas que te cuente una cosa? (Alonso de Santos J., Trampa para pájaros, 1990)

(3) AMA aparece, con todos sus velos y vestidos enlutados, despatarrada y sentada sobre un bidet, lavándose.

Se levanta y deja bajar las prendas, esponjándose como una gallina negra. (CREA: Riaza L., El palacio de los monos, 1982).

En el ejemplo (1) el causante se caracteriza por una actitud pasiva, o sea no impide que otro participante, aquí el perro Chitón, realice el proceso de entrar en el coche. La implicación del sujeto principal parece ser más activa en los demás casos: en (2) la actividad consiste en pedir permiso a otro participante para realizar el evento de contar algo. En el ejemplo (3) el sujeto $A M A$ es agentivo porque deja bajar voluntariamente las prendas, realizando movimientos apropiados. En justicia hay que admitir, como lo hace Silva, que los ejemplos que acabamos de ver son casos 'modelo' y que a menudo resulta difícil colocar una etiqueta inequívoca en la semántica de dejar, como en los casos siguientes:

(4a) Dicho lo cual, como el minuto y medio no le parecía suficiente para su ‘body', Gallardón ha optado por aparecer a través del circuito regional de TVE, cuyo director general José Antonio Sánchez le deja chupar más cámara, cuatro minutos exactamente. (CREA: La Razón digital, 19/12/2003) 
(4b) Ramón Pedrosa.- Quizá... (Deja la ironía y deja escapar tonos de rencor.) (CREA: Martín Recuerda J., Las arrecogías del beaterio de Santa María Egipcíaca, 1980)

Así resulta difícil determinar en qué medida el uso de dejar en (4a) implica una autorización explícita o significa más bien 'no oponerse a que Gallardón chupe más cámara'. En el ejemplo (4b) nos topamos con otro tipo de ambigüedad, ¿el verbo dejar significa 'hacer escapar voluntariamente unos tonos de rencor' o 'no oponerse a que salen involuntariamente'? Veremos efectivamente a lo largo del análisis que la polisemia de dejar es tal que muchas veces es imposible distinguir claramente los diferentes valores semánticos que menciona la literatura citada. Prescindiendo de los casos dudosos en nuestro análisis, reduciremos la complejidad semántica de dejar a una dicotomía esencial entre causación directa y autorización.

Al lado de eso, el estudio contribuye a una mejor comprensión de la interfaz sintaxissemántica a partir de un análisis empírico tanto cuantitativo como cualitativo ${ }^{1}$. Más concretamente intentaremos contestar a dos preguntas de investigación esenciales: (1) ¿los rasgos semánticos de los principales constituyentes de la construcción causativa - el causante S1, el causado S2 y el infinitivo - tienen o no un impacto en su comportamiento sintáctico? y (2) ¿Existe o no una relación entre estos rasgos y la polisemia de dejar causativo? Comprobaremos estas preguntas mediante el análisis sistemático de las variaciones sintácticas generalmente asociadas con la factitiva, a saber la selección de un complemento infinitivo o de una completiva (sección 1), la posición variable del causado relativamente al infinitivo (sección 2) y la marca acusativo o dativo del causado pronominal (sección 3) ${ }^{2}$. El análisis será

\footnotetext{
${ }^{1}$ El artículo actual se sitúa en la tradición de los estudios de corpus, que mediante el análisis detenido de un número considerable de ejemplos reales quiere examinar la actitud lingüística de locutores nativos. Nuestro corpus, compuesto a partir del Corpus de Referencia del Español Actual, contiene aproximadamente 1000 ejemplos de la construcción dejar + infinitivo (o completiva) con un participante subordinado nominal o pronominal, y tiende a ser representativo del español peninsular actual. Nos servimos además de un breve corpus de traducciones (las traducciones al español y al francés de la novela inglesa Harry Potter and the Sorcerer's Stone de J.K. Rowling, cf. bibliografía) para ilustrar o confirmar las conclusiones conseguidas.

${ }^{2}$ En el estudio actual no realizamos un análisis detallado del fenómeno de la subida de los clíticos, que el lector puede leer en Roegiest/Enghels (2009). Sin embargo, de ser útil establecemos una comparación entre los factores que influyen en esta variación sintáctica y los fenómenos aquí estudiados (cf. infra 3.2).
} 
multifactorial $^{3}$ y tendrá en cuenta el impacto de esquemas semánticos enteros en vez de las propiedades de los constituyentes individuales, por lo que se destaca también de aproximaciones anteriores.

\section{Construcción con infinitivo vs. construcción con completiva}

Ya sabemos que, contrariamente al francés, el español construye dejar causativo con un infinitivo o con una completiva (Enghels / Roegiest en prensa). La idea general que resalta de los estudios de Silva (1997, 2003), Paris (1999), Rodríguez Espiñeira (1999) o Maldonado (2007) es que la completiva se caracteriza por una relación e interacción menos directa entre el sujeto principal S1 y el causado subordinado S2, por lo que el sujeto subordinado tiene un estatuto más independiente y menos afectado en la completiva que en la factitiva con infinitivo.

\subsection{Selección del complemento y análisis multifactorial}

Con el fin de examinar en qué medida la selección del infinitivo o de la completiva depende de parámetros diferentes, realizamos un análisis multifactorial que implica los rasgos semánticos de los principales constituyentes de la construcción factitiva con dejar: el causante, el causado y el verbo subordinado. Tomamos en consideración el grado de dinamicidad potencial de esos constituyentes, o sea el carácter +/- animado de los participantes y el carácter transitivo, inergativo, inacusativo o pronominal del verbo subordinado.

Más particularmente, clasificamos los participantes según su capacidad o no de causar un cambio de estado físico o mental. Los humanos y animados que autocontrolan su actividad (p.ej. coches) son potencialmente dinámicos (indicados mediante [+ ANIM] en las tablas),

\footnotetext{
${ }^{3}$ Aunque no refiere a las técnicas estadísticas avanzadas como ANOVA o la regresión logística. Nótese además que como los datos estadísticos sirven solo como punto de partida del análisis cualitativo, prescindimos de incluir pruebas estadísticas que midan el grado de casualidad de las tendencias observadas.
} 
mientras que los inanimados no-autocontroladores (p.ej. casa) y los abstractos no lo son (clasificados como [-ANIM]). Los infinitivos se clasifican según el grado de transferencia de energía que implican (Enghels 2007). Los infinitivos transitivos (TR), de tipo comer, implican generalmente un alto grado de transferencia de energía entre dos participantes. Además, dentro del grupo de los intransitivos distinguimos los llamados 'inergativos' (INERG) de tipo bailar y los llamados 'inacusativos' (INAC) de tipo caer. Aquéllos representan una emisión de energía por parte de un participante dinámico, mientras que éstos implican una recepción de energía por un participante poco dinámico. Por consiguiente, en función de su grado de dinamicidad, los infinitivos se clasifican prototípicamente de la manera siguiente: TR > INERG > INAC. Finalmente, García (2009: 234) sostiene que la presencia de un verbo pronominal (PRON) aumenta el grado de autonomía del evento subordinado, por lo que lo consideramos también como un tipo separado pertinente.

De la tabla 1 se deduce que la combinatoria prototípica de la completiva comporta participantes animados y un verbo transitivo (5a), mientras que la combinatoria preferida de la construcción con infinitivo representa casi el polo opuesto: un argumento causado inanimado y un verbo intransitivo inacusativo (5b). Sólo el S1 es similar en ambas construcciones sintácticas:

(5a) Aunque bien puede decirse que todo lo motivó el navarro, que dejó que Olano se adjudicase un oro que él pudo y debió conseguir. (CREA: El Mundo, 30/10/1995)

(5b) Marina deja caer el jarrón donde puede y sale. (CREA: Bermejo A., E lucevan le stelle, 1992)

\begin{tabular}{|l|l|l|l|l|}
\hline \multirow{2}{*}{ esquema } & \multicolumn{2}{c|}{ INF } & \multicolumn{2}{c|}{ COMPL } \\
\cline { 2 - 5 } & \multicolumn{1}{c|}{$\#$} & \multicolumn{1}{c|}{$\%$} & \multicolumn{1}{c|}{$\#$} & \multicolumn{1}{c|}{$\%$} \\
\hline S1 [ANIM] + S2 [-ANIM] + V [INAC] & $\mathbf{1 6 9}$ & $\mathbf{4 3 , 1 \%}$ & 38 & $19,2 \%$ \\
\hline S1 [-ANIM] + S2 [-ANIM] + V [INAC] & 77 & $19,6 \%$ & 2 & $1 \%$ \\
\hline S1 [ANIM] + S2 [ANIM] + V [INAC] & 63 & $16,1 \%$ & 9 & $4,5 \%$ \\
\hline S1 [ANIM] + S2 [ANIM] + V [INERG] & 42 & $10,7 \%$ & 14 & $7,1 \%$ \\
\hline S1 [ANIM] + S2 [-ANIM] + V [INERG] & 17 & $4,3 \%$ & 6 & $3,1 \%$ \\
\hline S1 [ANIM] + S2 [ANIM] + V [TR] & 10 & $2,6 \%$ & $\mathbf{5 9}$ & $\mathbf{2 9 , 8 \%}$ \\
\hline S1 [ANIM] + S2 [ANIM] + V [PRON] & 4 & $1,1 \%$ & 18 & $9,1 \%$ \\
\hline S1 [-ANIM] + S2 [ANIM] + V [INAC] & 3 & $0,8 \%$ & 0 & - \\
\hline S1[ANIM] + S2 [-ANIM] + V [TR] & 3 & $0,8 \%$ & 26 & $13,1 \%$ \\
\hline
\end{tabular}




\begin{tabular}{|l|l|l|l|l|}
\hline S1[ANIM] + S2 [-ANIM] + V [PRON] & 2 & $0,5 \%$ & 25 & $12,6 \%$ \\
\hline S1[-ANIM] + S2 [ANIM] + V [TR] & 1 & $0,3 \%$ & 0 & - \\
\hline S1 [-ANIM] + S2 [-ANIM] + V [INERG] & 1 & $0,3 \%$ & 0 & - \\
\hline S1 [-ANIM] + S2 [ANIM] + V [INERG] & 0 & - & 0 & - \\
\hline S1[-ANIM] + S2 [ANIM] + V [PRON] & 0 & - & 0 & - \\
\hline S1[-ANIM] + S2 [-ANIM] + V [TR] & 0 & - & 1 & $0,5 \%$ \\
\hline S1[-ANIM] + S2 [-ANIM] V [PRON] & 0 & - & 0 & - \\
\hline TOTAL & 392 & $100 \%$ & 198 & $100 \%$ \\
\hline
\end{tabular}

Tabla 1. Tipo de complemento y esquemas semánticos

El análisis multifactorial muestra también una correlación entre la semántica del causante, el tipo de complemento seleccionado y la semántica del verbo principal. Generalmente se observa que con dejar causativo dominan los causantes animados: el 85,6\% (a saber 505 de los 590 casos en total) de los ejemplos analizados conlleva un sujeto principal humano (cf. ejemplos 7 infra). Este fenómeno se explica claramente por el significado de causación negativa de dejar: solo el causante humano puede dejar el control al S2 del evento causado. Sin embargo, el causante inanimado - si se presenta - desencadena casi siempre el uso del infinitivo: un 21\% (82 de los 392 casos) de dejar + inf tiene un S1 inanimado (ejemplo 6 infra); con la completiva un S1 inanimado es casi inexistente (3/198). Este comportamiento concuerda con la polisemia de dejar causativo: lo que posibilita la causación con un S1 inanimado es la propiedad inherente del S1 que por eso resulta dominante con respecto al S2, generalmente también inanimado y por consiguiente poco dinámico, lo que provoca generalmente el uso del infinitivo. Dejar significando 'posibilitar' se aproxima pues a la causación positiva, como se deduce del ejemplo siguiente:

(6) Al caer una primera canica en la caja del balancín inferior, empuja hacia arriba al dosificador que deja escapar una canica mientras retiene a las otras. (CREA: VV. AA., Tecnología, 1995)

La diferencia principal entre la construcción con infinitivo y la completiva parece situarse en el tipo de evento subordinado. Efectivamente, el análisis combinatorio de los parámetros mencionados confirma que la construcción dejar que $+V$ combina prototípicamente un S2 animado con un verbo transitivo (7a) o pronominal (7b), mientras que dejar + infinitivo 
combina un S2 inanimado no dinámico con un verbo inacusativo y un S1 animado o inanimado (7c), como lo ilustran los ejemplos siguientes:

(7a) Yo quiero hacerte dos preguntas. Luego dejaré que Pedro también haga las que quiera. (CREA: TVE 1, 24/09/1995)

(7b) Velázquez dejó que el conde se adelantara y oyó cerrar la puerta a sus espaldas. (CREA: Chamorro E., La cruz de Santiago, 1992)

(7c) Mary deja caer el arma al suelo y mece al niño, mirando con ojos angustiados el cuerpo de su marido. (CREA: Buero Vallejo A., La doble historia del doctor Valmy, 1976)

Estos datos corroboran que las completivas se relacionan con un grado mayor de autonomía potencial del evento causado y de dinamicidad del $\mathrm{S} 22^{4}$.

\subsection{Selección del complemento y polisemia de dejar}

Como mencionamos en la introducción, no siempre resulta evidente decidirse sobre el valor semántico exacto adoptado por dejar. Sin embargo, en cuanto a la medida en que la polisemia de dejar condiciona la selección de la construcción sintáctica, se notan - al lado de lo que hemos observamos en la sección anterior - algunas tendencias claras.

En general, como dejar admite completiva (contrariamente al laisser francés), la construcción infinitiva suele ocurrir cuando dejar es semi-auxiliar y fusiona con el predicado subordinado en un predicado complejo en el que el S2 se pospone al infinitivo. En este predicado complejo, el S2 asume la función sintáctica disponible: va marcado como OD, si el infinitivo es intransitivo y como OI si el infinitivo se acompaña de un OD propio ${ }^{5}$

\footnotetext{
${ }^{4}$ Nótese que la complejidad formal del S2 y del infinitivo también han sido considerados durante el análisis cualitativo de los ejemplos. Así notamos por ejemplo que la presencia de complementos del infinitivo contribuye a la posposición del infinitivo (a) o al uso de una completiva (b). Para más ejemplos véase (Enghels/Roegiest, en prensa):

(a) A los cuatro o cinco días se sustituyen esos cuadros por otros con láminas, se limpia y se desinfecta el fondo y se deja a la colmena seguir con su ritmo natural. (CREA: Albert Rubio J., Apicultura ecológica, 2002)

(b) [...] fue dando la mano a todos los que me precedían y al llegar a mí, no dejó que Felipe hiciera la presentación. (CREA: Feo J., Aquellos años, 1993)

5 De acuerdo con el llamado 'principio de la unicidad estratal' (stratal uniqueness law) de Comrie (1976). Volveremos infra a los pocos casos en que dejar y el infinitivo no fusionan y que el S2 se antepone al infinitivo.
} 
Particularmente cuando dejar se combina con una completiva, se observa que tiene una estructura argumental propia cuya complejidad parece relacionarse con su polisemia. Efectivamente, aparte de ser bivalente $(8 \mathrm{a}, 8 \mathrm{~b})$, se manifiesta también como trivalente, marcando un argumento como el OD y otro como el OI, equiparable con sus cuasi-sinónimos permitir o autorizar $(8 \mathrm{c}, 8 \mathrm{~d})^{6}$ :

(8a) Velázquez dejó que el conde se adelantara y oyó cerrar la puerta a sus espaldas. (CREA: Chamorro E., La cruz de Santiago, 1992)

(8b) Torija dejó que sus labios apuntaran una sonrisa. (CREA: Silva L., El alquimista impaciente, 2000)

(8c) Le dijo que su deber era salvarle el alma, y que si se ponía en orden con Dios le dejaba que le diera la teta a la niña. (CREA: Chacón D., La voz dormida, 2002)

(8d) Es como una policía del idioma, que lo tienen preso y no lo dejan que fluya y que vaya por la calle y se pervierta, que es lo que es estupendo de los idiomas vivos. (CREA: La vida según..., 24/09/95, TVE 1)

De estos ejemplos se infiere que dejar admite dos estructuras argumentales alternantes: una estructura con OD inanimado (el evento causado) o una estructura con OD animado (el argumento causado). Esta última configuración coincide con la sintaxis de los verbos de influencia, como autorizar (que admite la misma alternancia), permitir, invitar, etc. cuyo significado corresponde generalmente al de dejar en estas oraciones ${ }^{7}$. Un repaso del corpus de traducciones confirma en efecto que dejar con completiva corresponde a permettre en francés en 3 de los 5 casos que no aparecen con laisser:

(9a) But Dumbledore let me stay on as gamekeeper. (HP-PHST-IN: 46)

(9b) Pero Dumbledore dejó que me quedara como guardabosques. (HP-PHST-ESP: 61)

(9c) Mais Dumbledore m'a permis de rester comme garde-chasse. (HP-PHST-FR: 40)

\footnotetext{
${ }^{6}$ En el español peninsular el causado aparece sobre todo en dativo ( $l e$ vs. $l o=30$ vs. 8 casos), incluso en femenino, menos frecuentemente en el acusativo que refiere en la mitad de los casos a un inanimado. Cabe subrayar que la trivalencia sólo ocurre con un S1 humano.

${ }^{7}$ Citemos a título ilustrativo a Cuervo (2002) que confirma que permitir se construye a menudo con una proposición infinitiva o subjuntiva y un segundo argumento en dativo:

(a) Y si no te permiten hablarle, míralo hasta cansarte. Y cuéntanos mañana lo que has visto. (Neruda, Poesía, p 157)

(b) El señor Custodio la atajaba en seguida, sin permitirle que siguiere adelante. (Baroja, La busca 3.7, p 173)
} 
Por el otro lado sorprende el uso casi exclusivo de la completiva con verbos inacusativos como pasar y transcurrir acompañados de un S2 inanimado, un conjunto estructural que corresponde a los parámetros de la construcción con infinitivo y va en contra de los parámetros prototípicos de la construcción con completiva. Expresando el transcurso de un lapso de tiempo, ese dejar bivalente - diferente del dejar de permiso o de autorización - se aproxima al significado de 'esperar' como lo subraya el contexto de los ejemplos (10a-b):

(10a) Tengo que dejar que pase el tiempo, tengo que esperar...Vélez suspiró, tranquilizándose: si María dejaba que pasaran quince días, un mes o dos o tres, por mucho que dijera que quería volver, de hecho nunca volvería. (CREA: Pombo Á., El metro de platino iridiado, 1990)

(10b) Luego pasó educadamente la pipa al jipi del taburete contiguo, dejó que transcurrieran dos o tres minutos de procesión interior, [...]. (CREA: Sánchez Dragó F., El camino del corazón, 1990)

Por consiguiente, el estudio de la selección del tipo de complemento sugiere que dejar dispone de una estructura argumental compleja que refleja la polisemia del verbo a la vez que su integración en la categoría léxica de los verbos de influencia. A ver si los otros fenómenos sintácticos estudiados confirman esta hipótesis.

\section{Infinitivo con causado pospuesto o antepuesto}

Es sabido que también la posición (preverbal o posverbal) del causado tiene implicaciones semánticas. Conforme el autor, depende de la dinamicidad del causado (García 2009), de la causación directa o indirecta, o sea del grado de control por parte del causante (Treviño 1994), y o de la presencia de una o dos fuentes de energía (Silva 1997), lo que permite interpretar la construcción infinitiva como consistiendo en dos eventos concatenados o un solo hiperevento reducido (García 2009). 


\subsection{Posición del causado y análisis multifactorial}

El análisis multifactorial corrobora una oposición nítida entre la combinatoria de la construcción con S2 preverbal y con S2 posverbal:

\begin{tabular}{|c|c|c|c|c|}
\hline \multirow[t]{2}{*}{ esquema } & \multicolumn{2}{|c|}{ PO } & \multicolumn{2}{|c|}{ PREV } \\
\hline & \# & $\%$ & \# & $\%$ \\
\hline S1 [ANIM] + S2 [-ANIM] + Inf [INAC] & 169 & $45,3 \%$ & - & - \\
\hline S1 [-ANIM] + S2 [-ANIM] + Inf [INAC] & 77 & $20,6 \%$ & & \\
\hline S1 [ANIM] + S2 [ANIM] + Inf [INAC] & 62 & $16,6 \%$ & 1 & $5,3 \%$ \\
\hline S1 [ANIM] + S2 [ANIM] + Inf [INERG] & 37 & $9,9 \%$ & 5 & $26,3 \%$ \\
\hline S1 [ANIM] + S2 [-ANIM] + Inf [INERG] & 16 & $4,3 \%$ & 1 & $5,3 \%$ \\
\hline S1[ANIM] + S2 [ANIM] + Inf [TR] & 4 & $1,1 \%$ & 6 & $31,6 \%$ \\
\hline S1 [ANIM] + S2 [ANIM] + Inf [PRON] & 1 & $0,3 \%$ & 3 & $15,8 \%$ \\
\hline S1 [-ANIM] + S2 [ANIM] + Inf [INAC] & 3 & $0,8 \%$ & - & - \\
\hline S1[ANIM] + S2 [-ANIM] + Inf [TR] & 1 & $0,3 \%$ & 2 & $10,6 \%$ \\
\hline S1[ANIM] + S2 [-ANIM] + Inf [PRON] & 1 & $0,3 \%$ & 1 & $5,3 \%$ \\
\hline S1[-ANIM] + S2 [ANIM] + Inf [TR] & 1 & $0,3 \%$ & - & - \\
\hline S1 [-ANIM] + S2 [-ANIM] + Inf [INERG] & 1 & $0,3 \%$ & - & - \\
\hline S1 [-ANIM] + S2 [ANIM] + Inf [INERG] & - & - & - & - \\
\hline S1[-ANIM] + S2 [ANIM] + Inf [PRON] & - & - & - & - \\
\hline S1[-ANIM] + S2 [-ANIM] + Inf [TR] & - & - & - & - \\
\hline S1[-ANIM] + S2 [-ANIM] + Inf [PRO] & & 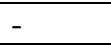 & - & - \\
\hline TOTAL & 373 & $100 \%$ & 19 & $100 \%$ \\
\hline
\end{tabular}

Tabla 2. Posición del causado y esquemas semánticos

Comprobamos (supra 1.2) que la construcción infinitiva con S2 antepuesto es excepcional y que entra en competencia con la completiva. Los datos confirman efectivamente la importancia de la dinamicidad inherente del causado y del evento subordinado. El 85\% (o sea 16 de los 19 ejemplos) de los causados antepuestos refieren a un participante inherentemente dinámico (11a). Incluso cuando el S2 antepuesto es abstracto, se concibe como fuente de energía (11b), sobre todo por el tipo de evento subordinado que encabeza:

(11a) Se parte del supuesto de que si se deja a las personas actuar en un clima totalmente informal y con absoluta libertad [...]. (CREA: Mora Mérida J.A. et al., Psicología aplicada a la actividad físico-deportiva, 2000)

(11b) [el hombre] se reconoce en cada forma de lo real y deja a la voluntad seguir su juego, pero sin colaborar con ella por medio de la afirmación despiadada de su singularidad egoísta. (CREA: Savater F., Invitación a la ética, 1982)

Paralelamente, el S2 antepuesto se combina con un infinitivo dinámico: la sola transitividad o inergatividad del verbo es un factor favorable a la anteposición del S2. 


\subsection{Posición del causado y polisemia de dejar}

En cuanto al impacto del significado del verbo, se nota que el S2 antepuesto siempre está marcado con la preposición $a$, incluso bajo condiciones menos propicios al uso de la preposición, como en (11b) y (12a-b):

(12a) A los cuatro o cinco días se sustituyen esos cuadros por otros con láminas, se limpia y se desinfecta el fondo y se deja a la colmena seguir con su ritmo natural. (CREA: Albert Rubio J., Apicultura ecológica, 2002)

(12b) En las generaciones siguientes dejaba a las plantas reproducirse naturalmente, sin temor a complicaciones provocadas por el polen proveniente de otra planta. (CREA: Fuentes Yagüe J.L., Iniciación a la botánica, 2001)

La ocurrencia general de la marca sugiere otra vez que el locutor toma como modelo la estructura argumental trivalente de los verbos de influencia como permitir y autorizar, que es también el significado que vehicula la gran mayoría de los ejemplos registrados.

Al mismo tiempo, la posposición del causado con dejar y un infinitivo transitivo sigue bastante frecuente:

(13a) Y deja saborear ese vino a esta servidora, que se le baje el ladrillo de empanada que tiene en la garganta. (CREA: Lázaro Maribel, Humo de beleño, 1986)

(13b) Y a los guerristas, a los renovadores, al señor Arzalluz cada vez que vaya a soltar otra coz verbal, a la panda de inútiles que han doblado la película Priscilla y, como un favor personal, a doña Cristina Almeida cuando vuelva a lo de Hermida, que la última vez no dejó decir ni mu al Mendicutti, oye. (CREA, El Mundo, $06 / 10 / 1994)$

Esta construcción convierte el S2 en OI, por lo que dejar, como semi-auxiliar con infinitivo, corresponde a la estructura bipredicativa trivalente de un verbo causativo léxico. Tal configuración supone una jerarquía participativa en que el S1 asume una coerción más directa y convierte al S2 en un benefactivo. La intervención dominante del S1 es preponderante en todos los casos registrados en nuestro corpus, lo que aproxima dejar a 'causar'. 
El ejemplo (14) permite ilustrar muy claramente la correlación entre la posición del S2 y la polisemia de dejar:

(14a) [...] el padre egoísta no deja al niño ver su programa preferido y éste se lo gana preparándole una merienda con el pan de molde anunciante. (CREA: León J.L., Mitoanálisis de la publicidad, 2001)

(14b) [...] el padre egoísta no deja ver al niño su programa preferido [...].

En (14a) dejar denota que el padre no autoriza el actuar del niño, que es agentivo (ver adopta el sentido activo de mirar), mientras que la posposición del causado en (14b) implica intervención activa (manipulación) del padre, ocultando o mostrando el programa (ver en el sentido de percibir), quitándole de esta forma al causado su responsabilidad y denota por lo tanto una cohesión más fuerte entre ambos predicados: dejar se aproxima al significado de ‘causar' y el predicado complejo equivale a un verbo causativo como mostrar.

De manera similar, como observamos supra (sección 1.1), con un S1 inanimado lo que posibilita la causación es la propiedad inherente del S1 que por eso resulta dominante con respecto al S2, generalmente también inanimado y por consiguiente poco dinámico. Tal configuración provoca siempre la posposición del S2 al infinitivo y dejar asume el significado causativo de 'posibilitar' (cf. supra, ejemplo 6).

\section{La marca casual del causado: acusativo vs. dativo}

Llegamos al último fenómeno morfosintáctico, a saber el caso del participante subordinado expresado mediante un clítico, que como ilustran los ejemplos siguientes, varía formalmente entre el acusativo (lo, los, la, las) (15) y el dativo (le, les) (16) ${ }^{8}$ :

(15) Tampoco su propio partido, que lo deja hablar con la convicción de que antes o después caerá en la maraña del régimen. (CREA: El País, 02/12/1988)

(16) ¡A la calle, fuera! - le dijo Eguren. La ira no le dejaba hablar, se sentía injustamente intimidado, avasallado. (CREA: Sánchez-Ostiz M., Un infierno en el jardín, 1995)

\footnotetext{
${ }^{8}$ Claro que en una variante dialectal leísta resulta difícil decidir de la función sintáctica de los clíticos que refieren a un antecedente masculino. Sin embargo, con las nociones de 'acusativo' y 'dativo' referimos exclusivamente a la forma de los clíticos y no a su función (OD u OI).
} 
Esta variación casual no ha pasado desapercibida en la literatura (cf. entre otros Treviño 1994; Davies 1995; Paris 1999; Rodríguez Espiñeira 1999; García 2009) y ha sido investigada desde dos puntos de vista principales, uno sintáctico y otro semántico. El primer planteamiento parte de la idea de una incorporación de ambos predicados. De acuerdo con este análisis, la marca de caso del causado se asigna en función del llamado 'principio de la unicidad estratal' (Stratal Uniqueness Law) y depende de la naturaleza del infinitivo subordinado (cf. también supra 1.2). De hecho, la idea de que una relación gramatical sólo puede establecerse una vez con el predicado (cf. Comrie 1976), tiene como consecuencia que el sujeto de un infinitivo intransitivo sea marcado como OD, con el caso acusativo, mientras que el sujeto de un infinitivo transitivo acompañado de su propio OD está marcado como OI, a saber, con el caso dativo. Cuando el infinitivo subordinado se acompañe de un OD y un OI, el causado será necesariamente marcado como complemento oblicuo, mediante el caso instrumental. La observación de que no pocas veces el español presenta un comportamiento 'disidente' en el sentido de que el $S_{2}$ puede cliticizarse tanto en acusativo como en dativo, con independencia de la naturaleza sintáctica transitiva o intransitiva del verbo subordinado, hizo necesaria una reconsideración del problema. Miremos algunos ejemplos de Treviño (1994: 53):

(17a) El gitano lo/le hizo comprar sus inventos.

(17b) Anastasia lo/le hizo trabajar duramente.

(17c) Fue eso lo que lo/le hizo caer.

Consiguientemente, la segunda línea de investigación adopta una perspectiva semántica y se centra en la oposición entre la causación directa e indirecta ${ }^{9}$. La idea es que el caso acusativo produce una interpretación de causación directa mientras que el dativo coincide generalmente con un menor grado de control por parte del causante, o sea con un mayor grado de independencia del participante subordinado. Veremos, sin embargo, que este principio tampoco se confirma enteramente con dejar, precisamente por su polisemia particular.

\footnotetext{
${ }^{9}$ Por 'causación directa' se entiende que el sujeto principal actúa directamente sobre el tema causado mientras que en la 'causación indirecta' no tiene control inmediato sobre él (Shibatani 1975).
} 
De acuerdo con el análisis que precede pasamos en el apartado siguiente a un replanteamiento del problema en términos multifactoriales que tendrá en cuenta el impacto de los diferentes esquemas semánticos que rige el verbo principal.

\subsection{Selección del caso: análisis multifactorial ${ }^{10}$}

Tomamos como punto de partida la hipótesis según la cual el comportamiento de los clíticos no es arbitrario sino que obedece a ciertas necesidades comunicativas y cognitivas, dependiendo sobre todo de las relaciones entre los diferentes participantes implicados en la construcción (cf. García 2009; Kemmer / Verhagen 1994: 137). En lo que sigue trataremos de demostrar que este planteamiento multifactorial también se confirma cuantitativamente y que se observan correlaciones entre el caso del causado y los esquemas semánticos que adopta dejar. Tenemos aquí las frecuencias del caso acusativo y dativo en todos los contextos semánticos posibles ${ }^{11}$ :

\begin{tabular}{|l|c|c|c|c|c|c|}
\hline \multirow{2}{*}{ esquema } & \multicolumn{6}{|c|}{ caso S2 } \\
\cline { 2 - 7 } & \multicolumn{2}{|c|}{ acusativo } & \multicolumn{2}{c|}{ dativo } & \multicolumn{2}{c|}{ TOTAL } \\
\cline { 2 - 7 } & $\#$ & $\mathbf{\%}$ & $\#$ & $\mathbf{\%}$ & $\#$ & $\mathbf{\%}$ \\
\hline S1[DIN] + S2 [DIN] + Inf [TR] & 1 & $2,2 \%$ & 45 & $97,8 \%$ & 46 & $100 \%$ \\
\hline S1 [DIN] + S2 [DIN] + Inf [INERG] & 26 & $27,7 \%$ & 68 & $72,3 \%$ & 94 & $100 \%$ \\
\hline S1 [DIN] + S2 [DIN] + Inf [INAC] & 47 & $50 \%$ & 47 & $50 \%$ & 94 & $100 \%$ \\
\hline S1[-DIN] + S2 [DIN] + Inf [TR] & 1 & $14,3 \%$ & 6 & $85,7 \%$ & 7 & $100 \%$ \\
\hline S1 [-DIN] + S2 [DIN] + Inf [INERG] & 6 & $23,1 \%$ & 20 & $76,9 \%$ & 26 & $100 \%$ \\
\hline S1 [-DIN] + S2 [DIN] + Inf [INAC] & 3 & $27,3 \%$ & 8 & $72,7 \%$ & 11 & $100 \%$ \\
\hline S1[-DIN] + S2 [-DIN] + Inf [TR] & - & - & - & - & - & - \\
\hline S1 [-DIN] + S2 [-DIN] + Inf [INERG] & - & - & - & - & - & - \\
\hline S1 [-DIN] + S2 [-DIN] + Inf [INAC] & 8 & {$[100 \%]$} & - & - & 8 & $100 \%$ \\
\hline S1[DIN] + S2 [-DIN] + Inf [TR] & - & - & - & - & - & - \\
\hline S1 [DIN] + S2 [-DIN] + Inf [INERG] & 6 & {$[100 \%]$} & - & - & 6 & $100 \%$ \\
\hline S1 [DIN] + S2 [-DIN] + Inf [INAC] & 55 & $93,2 \%$ & 4 & $6,8 \%$ & 59 & $100 \%$ \\
\hline
\end{tabular}

Tabla 3. Variación de caso y esquemas semánticos

Resulta claramente de la tabla que los causados inherentemente dinámicos - humanos, animados e inanimados dinámicos - llevan el dativo, de acuerdo con las condiciones de

\footnotetext{
${ }^{10}$ El objetivo de trabajar con el número de ejemplos más alto posible nos ha forzado a recurrir a bancos de datos electrónicos que, desafortunadamente, no destacan más variantes dentro de la variante peninsular. Además, solo tenemos en cuenta los clíticos que refieren a la tercera persona, ya que son los únicos que marcan morfológicamente la diferencia entre el acusativo y el dativo.

${ }^{11}$ A pesar del estatuto particular del caso dativo con los infinitivos transitivos (leísmo o dativo argumental, pues OI) no los excluimos del análisis que sigue, por la simple razón de que la presencia de un transitivo no siempre conlleva el uso del caso dativo y que por tanto los ejemplos pueden ofrecer informaciones valiosas sobre el impacto de la semántica en el caso.
} 
aparición del leísmo (cf. por ejemplo Fernández Ordóñez 1999). Las pocas excepciones que encontramos en nuestro corpus peninsular se explican por factores ajenos a la construcción que examinamos. Así por ejemplo todas las ocurrencias de le con referente inanimado aparecen en combinación con se impersonal, y como se impersonal se combina casi siempre con el clítico dativo ( $s e$ le vs. se lo $=25$ vs. 1 ejemplo), no lo tendremos en cuenta en este estudio.

Por el otro lado, si se tiene en cuenta la semántica del complemento infinitivo entero, los datos empíricos muestran una clara tendencia decreciente a seleccionar la marca dativo a partir de los complementos con infinitivo más dinámicos $\left[S_{2} D I N+I N F T R\right]>\left[S_{2} D I N+I N F\right.$ $I N E R G]>\left[S_{2} D I N+I N F I N A C\right]$ hasta los complementos menos dinámicos con un causado [DIN]. Compárense los casos siguientes:

(18a) Alberto se imagina que es el gran salvador de todos y sobrevalora sus posibilidades. Los otros le dejan manipular las cosas con demasiada facilidad y después se lanzan a un revanchismo peligroso. (CREA: Anónimo, Cómo resolver los pequeños conflictos en el trabajo, 1991)

(18b) Pero las radiaciones de longitud más corta (ultravioleta, X, gamma y cósmicos) no llegan a la Tierra. Afortunadamente para nosotros, porque son nocivas, la atmósfera que rodea nuestro planeta actúa de filtro, las absorbe y no las deja pasar. (Oliver J.Ma., Manual práctico del astrónomo aficionado, 1992)

Más particularmente, el análisis multifactorial arroja un resultado bastante consistente. La configuración $S 1+$ S2 inanimado + infinitivo intransitivo siempre ocasiona el uso del clítico acusativo, lo que corresponde a la configuración de una construcción transitiva causativa. Dejar asume en ese contexto su valor semántico (causativo) básico de 'causar' o 'posibilitar'. Al revés, la configuración $S 1$ hum $+S 2$ hum + infinitivo transitivo provoca siempre la marca dativo.

\subsection{S2 + infinitivo transitivo y comparación con la subida}

En efecto, se deduce de la tabla 3 que la configuración más propensa al uso del dativo contiene un S2 dinámico y un infinitivo transitivo. Dos factores simultáneos - uno sintáctico, 
otro semántico - pueden explicar esta correlación. La mera presencia de un verbo transitivo con OD propio lleva a marcar el S2 como dativo, si se acepta la tesis de la fusión bipredicativa (cf. supra). En tal caso, el dativo corresponde a la posposición del S2 nominal al infinitivo, que constituye una estructura bastante frecuente en español (un $42.9 \%$ en nuestro corpus), como ocurre en:

(19) ¿Cómo ves tú la repetición de la jugada? Pues que por fin le dejan coger su Chupa Chups a Aznar. (CREA: Protagonistas, 05/05/97)

Sin embargo, la correlación entre dativo y S2 posverbal no basta para explicar la presencia del dativo: el S2 antepuesto al infinitivo transitivo queda dominante (57.1\%, cf. supra tabla 2), mientras que el uso del clítico acusativo con el mismo infinitivo transitivo es casi inexistente en el español peninsular.

De manera similar se considera generalmente la subida de los clíticos como un indicio de una reducción clausal, por lo que con un infinitivo transitivo el pronombre S2 aparece automáticamente marcado como OI en dativo:

(20) Me contó que le habían prohibido tener las flores en el cuarto desde el atardecer hasta la madrugada, porque las flores, en la oscuridad, se comen el oxígeno del aire. Yo ya lo sabía, pero se lo dejé contar con todo lujo de detalles. (CREA: Mendoza E., La verdad sobre el caso Savolta, 1975)

Se ha demostrado ya que la subida de los clíticos tampoco es preponderante con dejar, en contraste con la factitiva con hacer (Roegiest / Enghels 2009:261-263). Se nota efectivamente que incluso cuando los pronombres se cliticizan al infinitivo, aparece mayoritariamente (25 vs. 8 casos) el clítico dativo en el español peninsular:

(21a) Tras esta reunión, Antxon consigue que los dominicanos le dejen contársela a la cúpula de ETA a la que indica: [...]. (CREA: Gurruchaga C., San Sebastián I., El árbol y las nueces, 2000)

(21b) Entre ellos, ustedes, que en mala hora vinieron y nos pagaron la hospitalidad dejando el país lleno de huevos de serpiente. Franco les echó a correazos y ustedes pusieron los huevos allí donde les dejaron ponerlos, aprovechándose de la hospitalidad de hombres de corazón generoso como nuestro generalísimo Trujillo. (CREA: Vázquez Montalbán M., Galíndez, 1985) 
Al mismo tiempo se nota que el significado de dejar puede - si bien no debe - diferir correlativamente con la subida o no de los clíticos. En las oraciones con subida (cf. 20), el causante se limita a una actitud pasiva, y no se opone al evento realizado por el argumento causado agentivo, como se deduce también del ejemplo siguiente:

(22a) Ah. Un encendedor. ¿De dónde lo has sacado? ¿Me lo dejas ver? (CREA: Marsé J., Rabos de lagartija, 2000)

$\mathrm{Al}$ revés en las oraciones sin subida $(21 \mathrm{a}-\mathrm{b}, 22 \mathrm{~b})$, dejar se acerca otra vez a un verbo de influencia ya que el causante más activo le autoriza al causado realizar el E2:

(22b) Cuando ponían en La Clave películas como ‘El increíble hombre menguante’ o 'La invasión de los ladrones de cuerpos' me dejaba verlas hasta el final. (CREA: La Luna del siglo XXI, 14/03/2003)

Es también lo que observamos en los dos casos en que aparece una marca dativo con referente femenino:

(23a) 'Pues ya sabes lo que tienes que hacer.' Belinha le pidió que, por lo menos, le dejase pensarlo. (CREA: Torrente Ballester G., Filomeno, a mi pesar, 1988)

(23b) Comieron en la cama, recostados sobre las almohadas, y aunque Maribel insistió mucho en que le dejara hacerla otra vez, él se limitó a sacudir la sábana de arriba un par de veces para desalojar las migas. (Grandes A., Los aires difíciles, 2002)

Paralelamente en las oraciones que incluyen un solo clítico, observamos que la autorización por parte del S1 confiere más autonomía a ambos eventos de la causativa. En principio, el uso (raro) de un S1 acusativo con un infinitivo transitivo debería implicar también que ambos eventos conservan cierto grado de autonomía, y por tanto que dejar asumiría el significado más 'fuerte' de autorización o de permiso, lo que se confirma en las oraciones siguientes:

(24a) Tienen que comprenderme, lo he hecho por mi madre. La necesidad la ha trastornado; se pasa todo el día sentada en la cocina mirando fijamente a la bombilla para ver si termina de quedarse ciega y la dejan vender cupones. (CREA: Caballero E., Squash, 1988)

(24b) Ni siquiera Judith, que parecía tan frágil y tan perdida, lo necesita. No, las mujeres son estresantes porque no lo dejan hacerse el héroe y salvarlas. (CREA: Beccaria L., La luna en Jorge, 2001) 
Incluso con un infinitivo intransitivo, el dativo queda dominante, aunque la tabla 3 muestra que su frecuencia baja proporcionalmente al grado de dinamicidad verbal (infinitivo TR > INERG > INAC) y que el uso del acusativo aumenta considerablemente con la menor dinamicidad del infinitivo.

\subsection{S2 + infinitivo intransitivo: el impacto de la polisemia de dejar}

Esos datos corroboran nuestra hipótesis de que aparte de la estructura sintáctica, la polisemia de dejar tiene asimismo un impacto en la selección de la marca: con un infinitivo intransitivo el factor sintáctico ya no influye en la selección de la marca dativo o acusativo: el S2 no puede asumir más que la función sintáctica de OD, que sea marcado por la forma del dativo le (el llamado leísmo), o por la forma del acusativo. Puesto que en el contexto del leísmo varios factores influyen en la selección de la marca, prescindimos de un enfoque estadístico global. Efectivamente, observamos que en cuanto dejamos la construcción con infinitivo transitivo, la frecuencia del acusativo se debe en gran medida al femenino, lo que perturba hasta cierto punto los datos estadísticos. Véase la tabla:

\begin{tabular}{|c|c|c|c|c|}
\hline \multirow{2}{*}{} & \multicolumn{2}{|c|}{ S2 masculino } & \multicolumn{2}{c|}{ S2 femenino } \\
\cline { 2 - 5 } & $\#$ & $\mathbf{\%}$ & $\#$ & $\mathbf{\%}$ \\
\hline acusativo & 23 & $18,3 \%$ & 42 & $\mathbf{8 5 , 7 \%}$ \\
\hline dativo & 103 & $\mathbf{8 1 , 7 \%}$ & 7 & $14,3 \%$ \\
\hline total & 126 & $100 \%$ & 49 & $100 \%$ \\
\hline
\end{tabular}

Por eso preferimos concentrarnos en los casos estadísticamente minoritarios, más peculiarmente los casos con le femenino cuando la es mayoritario en una configuración dada (3.3.1) y los casos con el masculino lo cuando le masculino es mayoritario (3.3.2). De esta forma esperamos desvelar una correlación entre la marca del S2 y el significado de dejar.

3.3.1 Aunque - como se puede esperar - los resultados no son absolutos, se nota una tendencia nítida en cuanto al impacto potencial del valor del verbo principal en el caso del causado. Por lo menos, en 4 de los 7 casos con leísmo femenino, la realización del evento causado (E2) depende de la autorización o del permiso del S1: 
(25a) Mi padre se murió muy joven, a los 33 años. Era carabinero, porque el padre de mi madre no le dejaba casarse con un militar. (CREA: El Mundo, 21/09/1996)

(25b) Odia a su padre porque no le deja salir a la calle en sujetador como Madonna. (CREA: Carbonell J., Apaga... y vámonos. La televisión: Guía de supervivencia, 1992)

(25c) Mitterrand tenía 'charme' (encanto) a ojos de las mujeres. Se fiaba de ellas, les dejaba entrar en parcelas de conversaciones y gestos que negaba a los hombres. (CREA: El Mundo, 09/01/1996)

(25d) Cáritas Ruanda ha intentado entrar, pero al no ser periodistas tampoco le dejan entrar. (CREA: Hora veinticinco, 06/11/96)

Con le masculino se nota menos correlación con el significado peculiar de dejar: por el uso generalizado de le para un S2 con referente humano masculino como marca por defecto, la polisemia de dejar (casi) no tiene impacto.

3.3.2 Al revés, en las oraciones con acusativo masculino, dejar se limita en casi todos los casos (19 vs. 4 casos) a expresar la causa negativa y el S1 adopta una actitud tal que su intervención sólo sirve para (im)posibilitar la realización del evento causado ${ }^{12}$ :

(26a) Tampoco su propio partido, que lo deja hablar con la convicción de que antes o después caerá en la maraña del régimen. (CREA: El País, 02/12/1988)

(26b) [...] intentó moverse, sin dejar de mirar a su mujer, que permanecía erguida, como un monumento de indignación y de odio; pero una mano se le quedó enredada en la mano de la muerta, firme y agarrotada, que le sujetaba sin soltarlo y parecía la manifestación de un juicio de Dios y que, a pesar de sus esfuerzos y de sus prisas, no lo dejaba escapar, ni siquiera para que huyera de nuestro estupor. (CREA: Egido L., El corazón inmóvil, 1995)

(26c) Moisés se debate con angustia en trance de ahogarse. El funcionario, al fin, lo deja respirar. (CREA: Sastre A., Revelaciones inesperadas sobre Moisés. A propósito de algunos aspectos de su vida privada, 1991)

No extrañará pues que el S2 de un infinitivo como caer nunca aparezca marcado como dativo, siempre como acusativo:

\footnotetext{
${ }^{12}$ En un 36\% (30 de 83 casos) de las construcciones con le (masc.) + Inf INTR, el verbo dejar tiene el significado de autorización vs. solamente un $17 \%$ de casos con lo (4 de 23 ejemplos).
} 
(27) Todos hemos oído las historias de estos chicos 'que caen por las grietas de la sociedad', aunque sería más correcto decir que los dejamos caer de nuestras manos. (CREA: El Mundo, 03/12/1995)

3.3.3 Finalmente, según nuestros datos, la sustitución de un $\mathrm{S} 1$ humano por un $\mathrm{S} 1$ inanimado (o no dinámico) apenas tiene impacto en la selección de la marca del clítico. Sólo con un infinitivo inacusativo, la presencia de un causante inanimado parece influir más en el uso del dativo que un causante humano (lo/le con un S1 humano $=48 / 52$ vs. lo/le con un S1 inanimado $=36 / 64$ ), pero la diferencia se desvanece cuando limitamos los datos al masculino. En contraste, con un S1 inanimado, el le femenino parece algo más frecuente, a la luz de los pocos ejemplos de los que disponemos:

(28a) Me lo dijo una vieja desdentada y cadavérica, a la que el asma, además de otras goteras, no le dejaba dormir por la noche y se pasaba las horas espiándole la respiración a las enfermas, [...]. (CREA: Egido L., El corazón inmóvil, 1995)

(28b) A la televisión la mujer le dedica menos tiempo, pues la absorbe demasiado y no le deja trabajar. (CREA: González Duro E., Las neurosis del ama de casa, 1989)

Esos casos excepcionales podrían explicarse por la tendencia general al leísmo con un S1 inanimado.

\section{Conclusión general}

El análisis que precede muestra indiscutiblemente que por un lado los rasgos semánticos de los principales constituyentes de la construcción causativa (S1, S2 e Inf) tienen un impacto en el comportamiento sintáctico de la construcción entera, pero también la polisemia de dejar influye hasta cierto punto en la estructura sintáctica.

En cuanto a los rasgos inherentes de los constituyentes, se observa claramente una interfaz entre determinados esquemas semánticos y ciertas estructuras sintácticas. Prototípicamente los mismos parámetros son favorables (1) a la ocurrencia de la completiva, de un S2 preverbal, la subida de los clíticos y el caso dativo por un lado y (2) a la selección del 
infinitivo, además con S2 posverbal, con clíticos aglutinados al infinitivo y el caso acusativo por el otro lado. En términos generales la primera serie se correlaciona con la presencia de un evento subordinado dinámico, o sea con un causado animado y un verbo (infinitivo o conjugado) TR o INERG, mientras que la segunda serie se correlaciona más bien con una semántica menos dinámica del evento causado, o sea con un causado frecuentemente INAN o ABSTR y muchas veces un infinitivo INAC. Nótese además que sobre todo la construcción con completiva indica que dejar en español no sólo es semi-auxiliar sino tambien verbo léxicamente pleno. En efecto, la completiva se usa en general cuando ambos eventos causativo y causado - se consideran como bastante importantes para que el locutor las considere como dos predicados.

Sin embargo, cabe añadir que si comparamos los cuatro fenómenos sintácticos observados entre sí, se nota que la dicotomía de la marca casual (dativo vs. acusativo) corresponde menos a parámetros de las demás variaciones analizadas. Esta observación se explica por el hecho de que el campo operacional del dativo es mucho más amplio en la construcción causativa (por lo menos en el español peninsular), porque la sola causación favorece la ocurrencia de la marca, a condición de que el referente sea animado (o más en general dinámico), el dativo utilizándose por ejemplo tanto en combinación con un S2 posverbal (29), donde se esperaría un acusativo, o cuando los clíticos quedan con el infinitivo (cf. supra, p.ej. 21):

(29a) Carlota en un rápido movimiento le abraza fuertemente y se arroja con él al suelo. Theo le deja jugar agresivamente a su mujer. (CREA: Amestoy Egiguren I., Ederra, 1982)

(29b) Lo del Málaga no se entiende. Pone las entradas a 200 euros y luego no le deja jugar a gusto al Madrid. (CREA: As, 22/09/2003)

Estas observaciones confirman en grandes líneas los análisis anteriores de la factitiva en general (regida por hacer, cf. Roegiest / Enghels 2008) por lo que se confirma dejar como un verbo causativo. 
Al lado de eso, la sintaxis de dejar está influida también por el significado que vehicula el verbo principal mismo. Si prescindimos de los casos que admiten varias interpretaciones, la estructura semántica de dejar comporta de manera esquemática dos valores fundamentales. Por un lado dejar expresa la posibilidad de causación: las propiedades del S1 inanimado o la actitud del S1 humano posibilitan (o impiden) la realización del E2 (evento causado), lo que implica cierto control o cierta responsabilidad por parte del S1, sin transferencia de responsabilidad a S2. Por el otro lado, la actitud del S1 humano es tal que autoriza o permite a S2 que realice E2. En este caso, hay transferencia de responsabilidad a S2. En el primer caso, estamos confrontados prototípicamente con una estructura transitiva reducida a un predicado complejo, con S2 pospuesto al infinitivo (intransitivo), pronombre en acusativo y pronombres aglutinados a dejar. En el segundo caso se observa una correlación con la selección posible de una estructura trivalente con completiva, un complemento infinitivo con S2 antepuesto (casi exclusivamente marcado de 'a'), la no subida de los clíticos y la marca del dativo (aunque en menor medida, como acabamos de ver).

\section{Bibliografía}

\subsection{Corpus}

[CREA]

Real Academia Española. Corpus de Referencia del Español Actual. http://www.rae.es/

$[\mathrm{HP}]$ Rowling, J.K. 1997. Harry Potter and the Philosopher's Stone. London: Bloomsbury.

- 1998. Harry Potter à l'école des sorciers. Trad. por Jean-François Ménard. Paris: Gallimard jeunesse.

- 1999. Harry Potter y la piedra filosofal. Trad. por Alicia Dellepiane. Barcelona: Círculo de Lectores. 


\subsection{Obras citadas}

Comrie, B., 1976. 'The syntax of causative constructions: cross-language similarities and divergences', in The grammar of causative constructions: a conspectus, ed. M. Shibatani (New York / San Francisco / London: Academic Press), pp. 261-312.

Cuervo, R. J., 2002. Diccionario de Construcción y Régimen de la lengua castellana (Barcelona: Herder).

Davies, M., 1995. 'The Evolution of the Spanish Causative Construction', Hispanic Review, 63: 5777.

Enghels, R., 2007. Les modalités de perception visuelle et auditive: différences conceptuelles et répercussions sémantico-syntaxiques en espagnol et en français (Tübingen: Niemeyer).

Enghels, R., Roegiest, E., 2007. 'Les pronoms clitiques et la réduction propositionnelle dans les factitifs français et espagnols', in Studii de lingvistică şi de filologie romanică : hommages offerts à Sanda Reinheimer Rîpeanu (Bucureşti: Editura Universităţii din Bucureşti), pp. 245-258.

- en prensa. 'Los verbos de causación negativa dejar y laisser: sintaxis y polisemia', in Aspectualidad, transitividad, referencialidad. Las lenguas románicas en contraste, ed. V. Bellosta von Colbe, M. García García (Berlin / New York: Mouton de Gruyter).

Fernández Ordóñez, I., 1999. 'Leísmo, laísmo y loísmo', in Gramática descriptiva de la lengua española, ed. I. Bosque, V. Demonte, (Madrid: Espasa-Calpe), pp. 1317-1393.

García, E., 2009. The Motivated Syntax of Arbitrary Signs: Cognitive Constraints on Spanish Clitic Clustering (Amsterdam: John Benjamins).

Kemmer, S. / Verhagen, A., 1994. 'The grammar of causatives and the conceptual structure of events' Cognitive Linguistics, 5:115-156.

Maldonado, R., 2007. 'Soft causatives in Spanish', in On interpreting Construction Schemas, ed. N. Delbecque, B. Cornillie (Berlin / New York: Mouton de Gruyter), pp. 229-260.

Paris, L., 1999. The Spanish causative construction 'hacer-infinitive'. A Role and Reference Grammar Description (ms. Buffalo). 
Rodríguez Espiñeira, M.J., 1999. 'Problemas de interpretación sintáctica en cláusulas con dejar + infinitivo', in Homenaxe ó profesor Camilo Flores, ed. X.L. Couceito et al. (Santiago de Compostela: Universidade de Santiago de Compostela), pp. 306-331.

Roegiest, E., Enghels, R., 2008. 'La reducción oracional en la construcción factitiva española', in Lenguas en diálogo. El iberorromance y su diversidad lingüística y literaria. Ensayos en homenaje a Georg Bossong, ed. H.J. Döhla, R. Montero Muñoz, F. Báez de Aguilar González (Frankfurt am Main: Vervuert), pp. 285-306.

- 2009. 'La posición de los clíticos en la factitiva española: un estudio comparativo hacer v. dejar', in El hispanismo omnipresente. Homenaje a Robert Verdonk, ed. R. De Maeseneer et al. (Brussel: University Press Antwerp), pp. 253-264.

Shibatani, M., 1975. A linguistic study of causative constructions (Bloomington: Indiana University Press).

Silva, A. Soares da., 1997. A semântica de deixar (ms. Universidade Católica Portuguesa).

Silva, A. Soares da., 2001. 'La structure sémantique de laisser dans les langues romanes', in XXIII Congreso Internacional de Lingüística y Filología Románica, ed. F. Sánchez Miret (Salamanca: Max Niemeyer Verlag), pp. 441-456.

Silva, A. Soares da., 2007. 'Verbs of Letting: Some Cognitive and Historical Aspects', in On Interpreting Construction Schemas: From Action and Motion to Transitivity and Causality, ed. N. Delbecque , B. Cornillie (Berlin/New York: Mouton de Gruyter), pp. 171-200.

Talmy, L., 2000. Toward a cognitive semantics. 1. Concept structuring systems (Cambridge: MIT Press).

Treviño, E., 1994. Las causativas del español con complemento infinitivo, (México: Colegio de México).

Verhagen, A., Kemmer, S., 1997. 'Interaction and causation: Causative constructions in modern standard Dutch', Journal of Pragmatics, 27:61-82. 